\title{
Continuidad Educativa y Fondos de Conocimiento de Familias Venezolanas en Chile
}

\section{Educational Continuity and Funds of Knowledge of Venezuelan families in Chile}

Macarena Lamas-Aicón *, Patricia Thibaut

Universidad Austral de Chile, Chile

\section{DESCRIPTORES: \\ Fondos de Conocimiento \\ Continuidades y discontinuidades educativas}

Relación familia-escuela Migración

Justicia social

\section{RESUMEN :}

En los últimos años ha aumentado el número de estudiantes extranjeros en establecimientos educacionales. Así, este estudio se pregunta por los Fondos de Conocimiento (FdC) de familias venezolanas en el sur de Chile, como por las continuidades/discontinuidades con las prácticas educativas de la escuela pública chilena. A través del uso de metodología cualitativa con enfoque etnográfico, se realizó un total de quince entrevistas a cinco familias venezolanas, además de dos entrevistas grupales al profesorado de las escuelas en las que los hijos e hijas de estas familias estudian. Los resultados obtenidos a través de análisis de contenido señalan: alta continuidad de las prácticas, medios y fines entre las familias y las escuelas; alta valoración por parte del profesorado hacia estas familias migrantes, en relación a los sofisticados FdC que poseen asociado a su capital cultural y a su buen desempeño escolar, en contraste con los escolares locales. Esto sugiere mayor continuidad entre hogares venezolanos y escuela que entre hogares chilenos y escuela. En este sentido, la adherencia a la justicia social pone en evidencia la necesidad de atender también a los contextos en los que los estudiantes locales se están desarrollando, considerando integrar sus propios FdC en los proyectos curriculares.

KEYWORDS:
Founds of knowledge
Educational
continuities and
discontinuities
Family-school
relationship
Migration
Social justice

\section{ABSTRACT:}

The number of foreign students in educational establishments has increased in recent years. In this context, this study asks about the Funds of Knowledge (FoK) of Venezuelan families living in the south of Chile, as well as about the continuities/discontinuities with the educational practices of the Chilean public school. Using qualitative methodology with an ethnographic focus, a total of fifteen interviews were carried out with five Venezuelan families, in addition to two group interviews with the teachers of the schools where the children of these family's study. The results obtained through content analysis indicate: high continuity of practices, means and ends between families and schools; high appreciation by teachers of these migrant families, in relation to the sophisticated Fok they possess associated with their cultural capital and their good school performance, in contrast to local schoolchildren. This suggests greater continuity between Venezuelan households and schools than between Chilean households and schools. In this sense, the adherence to social justice highlights the need to also attend to the contexts in which local students are developing, considering integrating their own FoK into curricular projects.

CÓMO CITAR:

Lamas-Aicón, M. y Thibaut, P. (2021). Continuidad educativa y fondos de conocimiento de familias venezolanas en Chile. Revista Internacional de Educación para la Justicia Social, 10(1), 41-54.

https://doi.org/10.15366/riejs2021.10.1.003

*Contacto: macarena.lamas@uach.cl

ISSN: 2254-3139

revistas.uam.es/riejs
Recibido: $\quad 3$ de agosto 2020

$1^{\text {a }}$ Evaluación: 1 de noviembre 2020

2a Evaluación: 19 de febrero 2021

Aceptado: $\quad 27$ de marzo 2021 


\section{Introducción}

En los últimos 15 años Chile ha experimentado un incremento de población migrante que, si bien es menor en comparación a los demás países de la OCDE, se ha convertido en un fenómeno estructural (Jiménez y Fardella, 2015; Villalobos et al., 2017). Según datos del Instituto Nacional de Estadística, en coordinación con el Departamento de Extranjería y Migración (INE y DEM, 2020), el porcentaje de personas extranjeras residentes en Chile representa el 7,8\% respecto del total nacional, siendo la comunidad venezolana la que encabeza la lista en un 30,5\% del total de extranjeros, seguida por Perú y Haití. En la escuela, cifras oficiales del Ministerio de Educación (MINEDUC, 2018), estiman que el 57, 5\% de los niños/as inmigrantes, estudia en Establecimientos Municipales, potenciando el incremento de la matrícula total Pública en un $3,5 \%$.

Los estudios en este contexto, muestra al profesorado actuando principalmente desde diversos enfoques para atender a la diversidad cultural. En este sentido, pueden utilizar estrategias para invisibilizar la diferencia, al percibirla como entorpecedora de los procesos educativos o, en otro extremo, normalizarla, pero sin desarrollar cambios significativos en las metodologías de aula (Jiménez y Fardella, 2015). En casos más extremos, se han documentado prácticas exclusoras que transgreden los derechos del estudiantado (Pavez, 2012; Tijoux, 2013) basadas en estereotipos y prejuicios que afectan directamente su rendimiento, la inclusión y la convivencia dentro del aula.

Sumado a ello, predominan en las escuelas el desarrollo de actividades que folclorizan las prácticas culturales de las familias migrantes, estrategias que, si bien visibilizan la diversidad, traen implícita la noción de diferencia en términos esencialistas del concepto de cultura, lo que llevaría a entender el conjunto de conductas e incluso el desempeño escolar como fruto de pertenecer a un determinado colectivo (Cerón et al., 2017; Lalueza, 2012). Para el caso chileno, esto se remonta a la internación de población afrodescendiente esclava (Duconge y Guizardi, 2014) y la explotación/sometimiento de la población indígena que habitaba el territorio en tiempos de la Colonia española (Contreras-Cruces, 2016).

En la actualidad, estudios recientes acusan la existencia de un curriculum educativo monocultural que funciona de modo prescriptivo para las escuelas. Desde una postura crítica señalan que el curriculum debe modificarse para alinearse a prácticas docentes reflexivas, que persigan la transformación de las desigualdades, la lucha contra los prejuicios, estereotipos y el reconocimiento de las identidades diversas, las etnias, la migración y afrodescendencia, avanzando hacia una educación intercultural y educación antirracista (Joiko y Vásquez, 2016; Marolla, 2019; Riedemann y Stefoni, 2015).

Es por todo ello necesario deconstruir para avanzar hacia una educación intercultural, desde el reconocimiento de las estructuras que les han marginado históricamente, haciendo posible el reconocimiento mutuo. Así, para la familia migrante, su historia familiar, el proceso migratorio, las expectativas puestas en la nueva escuela, sus propios recursos e intereses, repercuten en la forma y contenido de las relaciones que establecen con la escuela que, a su vez, también otorga una respuesta educativa desde su propia historia y desde factores macro culturales, principalmente históricos, políticos y económicos (Ratner, 2013). En consecuencia, se hace necesario otorgar respuestas situadas a las necesidades del estudiantado, incorporando desde el curriculum los aportes y conocimientos que esta diversidad otorga.

Este artículo aborda el caso de la migración venezolana en Chile, colectivo que además de ser el más numeroso, presenta características propias de una inmigración forzada (López et al., 2019). Se pretende dar cuenta de la complejidad a la hora de analizar las relaciones de continuidad/discontinuidad entre familia inmigrante y escuela. Para ello nos enfocaremos en las características específicas de estas familias integradas en escuelas públicas en la ciudad de Valdivia, al sur de Chile. Lo haremos desde la perspectiva de los Fondos de Conocimiento (FdC) que, como propuesta teórica y metodológica, pretende apartarse del discurso del déficit, posicionando los conocimientos, habilidades y destrezas de las familias como saberes válidos para la práctica escolar y para la mejora de la relación entre ambos contextos de desarrollo. Las preguntas que guían este trabajo son: ¿Cuáles son los FdC de las familias venezolanas? y ¿qué continuidades y discontinuidades se observan entre familia y escuela en escuelas públicas chilenas? 


\section{Continuidades y discontinuidades entre familia-escuela y el modelo educativo chileno}

Son varios los estudios que han puesto énfasis en las relaciones entre familias diversas culturalmente y escuela (Cole et al., 2014; Esteban-Guitart et al., 2012; Ogbu, 1987; Poveda, 2001; Vila, 1998). En síntesis, se plantea que aquellas familias en alta continuidad con la escuela, es decir, familias que comparten metas educativas con éstas, enseñan a sus hijos las conductas esperadas para dicho contexto, cumplen con las demandas de la escuela y contribuyen con los procesos socializadores que ahí se despliegan, son familias que logran una más rápida y mejor adaptación al contexto escolar. En contraste, aquellas familias que no comparten necesariamente los mismos valores, motivos y metas educativas con ésta, que utilizan incluso prácticas, medios y fines que entran en conflicto con lo esperado en los centros educativos, son familias que presentan alta discontinuidad y que, por tanto, presentarán problemas en cuanto a los procesos de adaptación e integración escolar.

Sin embargo, la idea de continuidad entre familia y escuela nos remite a la imagen de una familia "ideal" que comparte la cultura escolar o, en términos de Bourdieu (1986), que comparte el habitus de la clase media occidental, y lo emplea como si todos los niños tuvieran acceso igualitario a ello. En consecuencia, se hace necesario considerar el proyecto educativo ilustrado de progreso de la escuela occidental, a nivel general, pero también atender al enfoque educativo chileno, en lo particular, que fija los criterios para valorar dicha continuidad.

Chile detenta un modelo de rendición de cuentas por desempeño escolar (Falabella y De la Vega, 2016) que, como varios autores señalan, ha derivado en la instalación en la escuela de lógicas del libre mercado (Bellei, 2010; Biscarra et al., 2015; Fardella, 2013). La definición de calidad educativa se ha aproximado al cumplimiento de protocolos y estándares de aprendizaje y desempeño en todas las áreas de la gestión escolar y particularmente reflejada en los resultados del estudiantado en los sistemas nacionales de evaluación (SIMCE y PSU). A esto se suma un sistema de voucher educativo que consiste esencialmente en subsidiar la demanda (estudiantes) y no la oferta (escuelas). Así, cada niño/a que ingresa a una escuela, lo hace con un determinado monto de dinero asignado en función de la asistencia a clases (Sandoval y Lamas 2017). Se piensa que las escuelas se esforzarán por mejorar su calidad educativa y, de esta manera, serán elegidas por las familias aumentando su matrícula y, por tanto, su financiamiento.

El modelo antes señalado recompensa a las escuelas con mayor asistencia a clases y con mejores resultados académicos. Esto pude ir en detrimento de sectores más vulnerables de la sociedad que, por múltiples factores y condiciones estructurales y sistémicas, presentan baja participación en la escuela (Gubbins y Otero 2016).

\section{Fondos de conocimiento como estrategia de inclusión escolar centrada en los recursos de las familias}

En alternativa a la perspectiva del déficit educativo y cultural, la aproximación de los FdC, ofrece una propuesta novedosa y de valoración de las prácticas educativas y de desarrollo que se originan al interior de las familias y de sus comunidades. Una de las tradiciones teóricas que respalda los FdC es la perspectiva sociocultural. La investigación en esta línea ha observado los procesos de desarrollo de las personas desde el nacimiento a la madurez. Estos estudios son interesantes para entender de qué manera las comunidades aseguraban que sus integrantes tuvieran oportunidades para participar y desarrollarse en actividades valoradas en su propio contexto de desarrollo (Rogoff, 2003; Rogoff y Lave, 1984; Scribner y Cole, 1973) y sirven para comprender el aprendizaje más allá de las instituciones formales. Además, esta perspectiva da cuenta de la variación que existe respecto a las expectativas de desarrollo, comportamiento y valores dependiendo de la cultura en la cual se esta inserto. Esto es relevante, en tanto muestra la diversidad existente en los procesos de desarrollo, así como en las variaciones de las expectativas educativas dependiendo del contexto cultural. Lo normativo es fruto de una aceptación de modelos que han sido consensuados, al menos tácitamente en una comunidad dada. Todo ello es importante de considerar a la hora de observar las continuidades y discontinuidades que se observarán más adelante en el análisis, así como el contexto escolar de llegada de los estudiantes. 
La premisa de los FdC es que todas las familias acumulan Fondos de Conocimiento, es decir, acumulan saberes, conocimientos, habilidades y destrezas que despliegan a través de sus particulares formas de vida: sus trabajos; ocupaciones; experiencias profesionales; redes de intercambio y ayuda mutua (Llopart y Esteban-Guitart, 2016; Moll et al., 1992). Esta acumulación de experiencias y aprendizajes situados es lo que constituye los Fondos de Conocimiento, entendidos como recursos con los que cuentan las familias para su desarrollo. El problema se sitúa cuando la escuela desconoce estos conocimientos, los invisibiliza o bien devalúa, al no formar parte del lenguaje hegemónico de la institución escolar (Iglesias et al., 2020; Poveda, 2001).

El proyecto original de FdC, utiliza la entrevista etnográfica, a través de visitas semanales a los hogares de las familias, como forma de acceder a los FdC que son documentados por los propios maestros y maestras del estudiantado migrante, quienes luego utilizaban esta información en el diseño de actividades curriculares que los considerarán. La pauta original de la entrevista trata temas tales como: historia migratoria, familiar y laboral; actividades cotidianas y educativas en el hogar, actitudes parentales, religión y economía. Los investigadores reconocen como elemento central en las prácticas familiares y comunitarias, la solidaridad, el intercambio y la reciprocidad, lo que contribuye a la construcción de confianza mutua como pegamento que une el tejido social que se crea (Llopart y Esteban-Guitart, 2016; Moll et al., 1992).

A través de los años, investigaciones basadas en FdC en diversos países y contextos, han evidenciado mejoras en las relaciones entre las comunidades de las familias migrantes y la escuela, puentes de conexión entre la vida de los estudiantes y los modelos instruccionales, al considerar de manera central la especificidad de contexto y el carácter situado de los aprendizajes (Esteban-Guitart et al., 2012; Esteban-Guitart y Saubich, 2013; González y Moll, 2002).

\section{Método}

El reporte de investigación que aquí se presenta, es parte de un proyecto mayor, inspirado en la estrategia de los FdC original, a decir: documentación de los FdC de las familias; diseño, ejecución y evaluación de actividades de aula basadas en los FdC detectados. En el presente reporte mostraremos la primera etapa del proyecto cuyos objetivos fueron: a) Identificar y describir los FdC de las familias migrantes venezolanas; b) analizar las continuidades y discontinuidades entre las familias migrantes y las escuelas.

Realizamos una investigación cualitativa de diseño de casos múltiples (Stake, 1995), y muestreo intencionado que consideró a cinco familias de origen venezolano pertenecientes a una escuela y un instituto Municipal de la Comuna de Valdivia, como lo señala el Cuadro1.

Cuadro 1

Características de las escuelas

\begin{tabular}{lcc}
\hline & Escuela (Es) & Liceo (Li) \\
\hline \% IVE & $93 \%$ & $91 \%$ \\
Promedio estudiantes por aula & 25 & 39 \\
Profesorado entrevistado & P1; P2; P3; P4 & P1; P2 \\
Familias participantes & F1; F2; F3 & F4; F5 \\
\hline
\end{tabular}

Se eligió a la escuela ya que reunía el mayor número de migrantes registrados en la zona, según datos ofrecidos desde la administración municipal. El Liceo, se incorporó luego del primer sondeo entre escuelas, a pesar de no contar con una alta cantidad de familias migrantes, pero si voluntad y disponibilidad por participar. Ambos centros escolares, reciben estudiantado con alto índice de vulnerabilidad escolar (IVE) de acuerdo Sistema Nacional de Asignación con Equidad. Cada familia fue considerada como un caso, siendo las prácticas (actividades) educativas que se desarrollan en su dinámica interna, la unidad de análisis. Fueron convocadas desde el establecimiento educacional correspondiente, utilizándose el criterio por conveniencia y voluntariedad. También se incluyó en el análisis al profesorado que dicta clases a los niños/as de estas familias. Para resguardar 
los aspectos éticos se garantizó la confidencialidad de la información, a través de consentimiento informado (López-Calva, 2019).

Como principal aproximación metodológica se utilizó las visitas etnográficas a los hogares de las familias. Sin embargo, se efectuó una modificación sustancial respecto al diseño original de los FdC, por cuanto, no incorpora las visitas del profesorado a los hogares de las familias, sino que éstas las realizan las investigadoras. Como técnicas de recolección, se utilizó la multi-metodología autobiográfica extendida (MMAE) (Peña-Cuadra y Esteban-Guitart, 2013), seleccionando las técnicas: entrevista en profundidad; construcción y exploración del genograma familiar y análisis de fotografías. Previa a la visita tres, se le daba a cada familia la indicación de tomar fotografías en donde pensaran que enseñaban algo a los niños y niñas. Cada encuentro tuvo un promedio de una hora y treinta minutos de duración. Las entrevistas se desarrollaron a través de tres grandes ejes indagatorios: Trayectoria educativa y laboral de las familias; relación familia-escuela; y prácticas educativas cotidianas en el hogar.

Con el profesorado, se realizó una entrevista grupal en la cual participaron cuatro profesores/as en el caso de la escuela y dos en el liceo. En este encuentro se pretendió hacer emerger los principales tópicos que permitan acceder a las continuidades y discontinuidades entre familia y la escuela, el conocimiento y la valoración que hacen de los saberes de las familias y la relación interactiva de aula.

La información fue sometida a análisis de contenido, identificando códigos emergentes que fueron contrastados entre sí hasta la saturación de información (Nascimento et al., 2016). Éstos, fueron agrupados en categorías temáticas que responden a los objetivos de esta investigación. Por último, el principal criterio de rigor científico fue la triangulación a través de la contrastación entre las investigadoras, la teoría de base y los datos obtenidos en la producción de la información (Johnson-Mardones, 2017).

\section{Resultados}

Uno de los primeros resultados es la descripción de las familias como se sintetiza en el Cuadro 2. La presentación de los demás resultados se organiza en torno a tres ejes temáticos: 1) Trayectoria educativa y laboral de padres, madres, abuelos y abuelas; 2) Prácticas educativas y de desarrollo; 3 ) Relación familia y escuela. Estos ejes se corresponden con nuestras categorías principales y temas de indagación, cada una de estas dimensiones contiene a su vez subcategorías para presentar los resultados.

Cuadro 2

Caracterización Socioeducativa y sociofamiliar

\begin{tabular}{|c|c|c|c|c|c|}
\hline & $\begin{array}{l}\text { Situación } \\
\text { conyugal }\end{array}$ & $\begin{array}{l}\text { Cantidad } \\
\text { hijos/as }\end{array}$ & Estudios padres & Ocupación actual & Religión \\
\hline $\mathrm{F} 1$ & Casados & 3 & $\begin{array}{l}\text { M: méd. veterinaria } \\
\text { P: méd. } \\
\text { traumatólogo }\end{array}$ & $\begin{array}{l}\text { M: Labores del hogar } \\
\text { P: méd. Gral. }\end{array}$ & Católica \\
\hline $\mathrm{F} 2$ & Divorciados & 1 & M: ingeniero & $\begin{array}{l}\text { M: profesora de mate- } \\
\text { máticas }\end{array}$ & Evangélica \\
\hline F3 & Casados & 1 & $\begin{array}{l}\text { M: periodista } \\
\text { P: educ. media }\end{array}$ & $\begin{array}{l}\text { M: Labores del hogar } \\
\text { P: Obrero en minera }\end{array}$ & Católica \\
\hline $\mathrm{F} 4$ & Casados & 2 & $\begin{array}{l}\text { M: antropóloga } \\
\text { P: abogado }\end{array}$ & $\begin{array}{l}\text { M: comercio ambulan- } \\
\text { te/labores del hogar }\end{array}$ & Católica \\
\hline F5 & Casados & 3 & $\begin{array}{l}\text { M: técnico en electrónica } \\
\text { P: méd. ginecólogo }\end{array}$ & $\begin{array}{l}\text { M: Labores del hogar } \\
\text { P: méd. Gral. }\end{array}$ & Católica \\
\hline
\end{tabular}

\subsection{Trayectoria educativa y laboral de padres, madres, abuelos y abuelas}

Esta categoría da cuenta de la estructura familiar, trayectoria laboral y escolar de los padres, madres, abuelos y abuelas, desde la perspectiva de las personas entrevistadas. Como se aprecia en la tabla dos, 
estas familias se caracterizan por contar con un nivel académico alto de padres y madres, lo que alude a su condición de profesionales con grado de educación superior universitaria, expectativas y exigencias que transmiten a sus hijos e hijas.

Obviamente hablando del punto de vista académico...eso es súper importante ¿no? Tienen que tener una buena formación académica para que ellas puedan de una forma más sencilla, optar por una carrera universitaria. Académicamente, yo pido todo, todo lo que puedan. (F1_M)

yo le digo a él "usted no puede ser menos que nosotros que teníamos menos", no teníamos nada y él tiene todas las oportunidades del mundo aquí, yo le digo "tú eres dueño de tu futuro $y$ el futuro de tus hijos", porque si no estudia, estamos a un nivel competitivo, donde entre más días, las cosas van a ser más difíciles. (F3_M)

Los oficios y ocupaciones de los adultos mayores de estas familias transitan desde médico cirujano, comerciante, agricultor, en el caso de abuelos a, en su mayoría, tareas domésticas para las abuelas. En todos los casos, las expectativas ilustradas de progreso, se transmiten como metas transgeneracionales.

mis padres son médicos y jamás me dijeron "estudia medicina" jamás me canalizaron en ese sentido, si insistían un poco más en el aspecto de "tienes que estudiar una carrera". (F5_P)

Y bueno de parte de mi esposo, el viene de una familia bien humilde, pero también lo impulsaron a estudiar, ellos a sus hijos los impulsaron que tenían que estudiar, que tenían que sacar una carrera. (F4_M)

Para el profesorado, el alto nivel educativo de los padres y madres es una ventaja por sobre las familias locales. Parecen poseer una mejor composición familiar, en comparación con los niños/as locales que, de acuerdo a lo relatado por el profesorado, vive en su mayoría en entornos precarizados y disfuncionales

Por lo menos mi alumna, el papá es doctor... vive con toda su familia, se vino toda su familia de Venezuela, están todos acá, tienen un hogar constituido o sea mamá, papá, hermanos, yes diferente a las familias a las familias de mis otros alumnos. Tengo muchos alumnos que viven solamente con la mamá... (Es_P1)

la mayoría de mis chicos (alumnado local) vienen de familias disfuncionales, hay algunos que ni siquiera tienen a los papás, solamente viven con la hermana, otros que viven solo con los abuelos, otros solo con la abuela, entonces hay un abismo gigante. (Es_P2)

\subsection{Prácticas educativas y de desarrollo}

Esta categoría da cuenta de las principales metas de desarrollo para los hijos e hijas. Se entenderá como desarrollo los cambios esperados en el sujeto, fruto de participar en prácticas socioculturales específicas, donde se definen metas y herramientas mediadoras para alcanzar dichos fines, a la vez que se desarrollan competencias y habilidades particulares que encuentran sentido y significado en la cultura. También forman parte de esta categoría los ritos y rutinas diarias en el hogar y la religión que profesan.

llega de la escuela y después de descansar un rato se pone hacer las tareas y generalmente se le revisa las tareas, o se ve lo que está haciendo, casi siempre la mamá que pasa más tiempo con ella, pero cuando ya tiene alguna dificultad con la evolución de la tarea, no entiende o necesita apoyo del desarrollo de algún tema, nosotros le damos el apoyo, mi esposa en la parte de matemática, química, física, esas cosas y yo en la otra parte donde yo me manejo de teoría, biología, ciencias, etcétera. Esa es más o menos la rutina de ella cuando llega. (F5_P)

La escuela ocupa un lugar central dentro de la dinámica interna y rutinas de las familias. Todo ello, repercute en que las prácticas de crianza se vinculen directamente con la etapa escolar, por ejemplo, establecer horarios para cada actividad, prestar ayuda en las materias escolares, guiar en la resolución de tareas escolares.

Le reviso sus cuadernos, si tienen alguna nota, pero ella ya normalmente me ha dicho:

"mamá tengo examen de no sé qué." Me dice todo, la grande es como más distraída, entonces 
tengo que revisarle, pero a ella ya no le reviso tanto, como cuando estaba más pequeña, si no que trato de dejar que ellas solas... a veces sé que tienen algo, porque tenemos esos grupos de WhatsApp". (F1_M)

"papito usted está encaminado, échele usted solo, porque usted, si algo no entienda, pregunte y si yo tampoco sé, vamos a buscar un tutorial en internet y revisamos", pero él está práctico en eso, busca un tutorial y entiende al tiro (enseguida), pero yo lo críe muy independiente, no puede estar esperando a que yo llegue para hacer algo". (F3_M)

Se valora la autonomía y responsabilidad personal por el éxito académico, definiendo como función principal de los niños/as en la etapa escolar, su rol como estudiantes.

Como yo siempre le he dicho, hay dos cosas nada más que tú tienes que hacer: a estudiary sacar buenas notas. Porque prácticamente no hace nada. Mamá lava la ropa, mamá cocina, mamá te atiende, mamá te saca donde tú quieres, mamá te lleva, mamá te trae, mamá te complace en todo. Entonces, ¿cuál es la recompensa a mamá y a ti mismo como persona? Estudiar. Estudiary sacarse buenas notas. Que van de la mano. Eso es lo único que quiero. (F2_M)

Además, se establece como herramienta para alcanzar dichas metas, el hábito de la lectura, en el sentido de reproducirse como una práctica social y experiencia formativa desde la temprana infancia.

arriba en su habitación, siempre intento actualizar un poquito los libros, le compro alguna cosa y ahí van leyendo todo el tiempo, por lo menos un libro que hay que estar leyéndole. (F2_M)

lo que pasa es que la lectura la hemos compartido siempre, de que son niños, yo te conté la otra vez desde que son niños, no sabían ni hablar, le leíamos cuentos, su papá y yo nos turnábamos para leerles cuentos, siempre, siempre, la lectura giró en torno a nuestras vidas. (F4_M)

En enlace con lo anterior, para las familias resulta crucial el fomento de la comunicación, específicamente relativa al uso del lenguaje, como forma de argumentar, explicar, reflexionar y, la conducta manifiesta, como herramienta de aprendizaje vicario.

(...) Trato de conversar. Sí, porque creo que regañándola no voy a lograr nada, solo que voy a hacer es como bloquearla, creo, no sé. A mí no me hubiera gustado que mi padre me enseñara algo que estuviera mal regañándome. Creo que sí me lo explicaba "oye esto pasa, esto te va a traer como consecuencia esto", yo creo que lo va a entender mejor. (F2_M)

lo que ellas copian no es lo que yo les digo que hagan, sino lo que ellas ven que yo hago (...) bueno uno a veces comete errores, uno no es perfecta, por supuesto y uno también se revisa y bueno a partir de alli, trato de que ellas, me copien. (F1_M)

Otro aspecto importante en la crianza, es que aprendan a incorporar en sus conductas el valor del respeto, sobre todo hacia los adultos. Se observa la posición que ocupa la niñez en la sociedad que deja entrever la jerarquía del mundo adulto, aspecto también reforzado por la religión que profesan.

Entonces creo que eso es parte de que los niños tienen que aprender que hay un respeto, que no significa sumisión, ni temor, pero si que hay un dialogo de respeto, que tienen que respetar a sus padres, que tienen que respetar a los adultos y a la gente que está alrededor, que cuando tienen alguna disconformidad que lo manifiesten, pero con respeto. (F5_P)

yo le digo a él, tiene que creer en un ser superior y ese valor creo que es el más importante. El que a uno lo eduquen con temor a alguien superior, con temor a Dios, es bueno, porque a veces uno evita de hacer tantas cosas, porque tiene temor a Dios. Y nosotros vamos a una iglesia cristiana. (F3_M)

Estas conductas y comportamientos también son reconocidos y valorados por el profesorado, destacando el aporte que ha significado la introducción de estos estudiantes en las aulas. 
tenemos niños mexicanos, de Venezuela, Colombia y son niños que son muy dulces, son niños que son muy educados que a mi me sorprende, que tiene un vocabulario mucho más rico que nuestros alumnos, entonces insisto que ellos han sido un tremendo aporte (...) son todos niños muy sanos, muy buenos, de familias muy comprometidas, que están siempre presentes en la escuela, entonces ellos han sido un tremendo aporte para el resto de sus compañeros". (Es_P4)

Respecto a ritos y ceremonias, las familias celebran los cumpleaños de sus integrantes y festividades como el día de la madre, del padre. Otros ritos que forman parte de la rutina son las actividades propias de la iglesia, como orar, celebrar semana santa y navidad, asistir a misa, preparar a los hijos/as para la comunión. Aún, cuando no todas las familias expresan ser practicantes, todas ellas adhieren al cristianismo y otorgan a la iglesia, la cualidad como lugar de encuentro y red de apoyo en el nuevo país.

ella ocupa mucho los fines de semana, ella ocupa mucho también con la iglesia, entonces el sábado va a la iglesia, el domingo va a la iglesia, y yo no voy a la iglesia, voy muy poco, hemos hecho amistad con los padres, por contactos a través de la iglesia donde ellos van y yo voy poco a la iglesia, todos somos católicos, pero yo soy poco asistente a la iglesia. (F5_P)

Todo lo anterior contrasta con lo que viven a diario los niños y niñas del país. Desde el reporte del profesorado, la distancia entre las experiencias vitales y rutinas diarias de los inmigrantes venezolanos con los primeros, es significativa.

el tema es complejo en cuanto a la experiencia de vivir en ese sitio, ellos lo comentan mucho, ellos ya se acostumbraron a dormir en la noche con balazos todos los días, entonces es bastante complejo la forma de vivir de ellos (...) hay problemas igual entre familias. (Es_P3)

\subsection{Relación familia-escuela}

Esta categoría contiene las formas de comunicación entre familia y escuela, la percepción de apoyo y expectativas mutuas, así como los roles y funciones definidos para cada contexto. Un primer elemento que se observa, son los ajustes en la dinámica interna de las familias producto de la migración, siendo el cambio en las ocupaciones y roles de los adultos, el más evidenciado.

bueno, tengo a mi esposo ahorita que él sostiene este hogar, económicamente hablando, súper trabajador (...) En la actualidad, (...) me estoy dedicando desde que llegué aqui a mis hijas, en este caso me corresponde a mi encargarme de todo lo que es el hogar, las niñas... cuento viejo, la mamá en la casa, el papá trabajando... no era común, pero desde que llegamos acá... (F1_M)

casi siempre va la mamá a las reuniones porque es la que está más acá, es la que tiene más disponibilidad y siempre ha sido así, yo siempre estoy muy ocupado con el trabajo. (F5_P)

En cuanto a las expectativas mutuas, para las familias la escuela cumple una función importante en la acogida y adaptación de sus hijos/as en el nuevo país, además de su función como agente socializador, en estrecha colaboración con la familia. Este aspecto es corroborado por el profesorado que percibe a las familias extrajeras como aliadas de los procesos educativos que ahí se detentan.

creo que la crianza es primordial, lo fundamental, la escuela inculca valores en buena medida, pero el tema fundamental es de los padres, participar con ella en las tareas de escuela, en sus actividades. (F5_P)

son familias muy comprometidas con los chicos (...) principalmente en lo que es tareas, o trabajos, presentación (...) asistencia a clases, o sea ahi se ve un compromiso de la familia, (...) ellos igual son súper abiertos a expresar lo que les pasa, cuando hay algún problema al tiro (enseguida) llegan los papás extranjeros, lo que no hacen los otros. (Es_P1)

En general, profesorado expresa altas expectativas hacia al desempeño académico del estudiantado inmigrante, lo cual asocian directamente con el capital sociocultural de sus familias de origen. No obstante, es importante recalcar que los docentes mencionan una diversidad en los estudiantes migrantes que han recibido y reportan esfuerzos para trabajar con todos los estudiantes independiente de su origen. 
el alumnado inmigrante va mucho más rápido en términos académicos que el alumnado de la escuela, esto porque presentan un nivel cognitivo alto. Además, existe alta expectativa tanto en habilidades sociales como en habilidades académicas, lo cual se espera ya que los padres tienen un nivel educativo muy alto, algunos con postgrados. (Li_P2)

Es un factor suerte, porque yo el año pasado tuve un alumno colombiano ... ahí la experiencia fue totalmente distinta... por la problemática que él tenía, era un niño como agresivo, intolerante a la frustración, y después se fue acentuando porque al comienzo como que no anduvo... pero ya después peleas, pateaba a la tía, a mi tía asistente. (P4, EF)

Otra dimensión asociada al alumnado local que se desprende de las entrevistas con el profesorado se relaciona con elementos disruptores, los cuales se reflejan en conductas que impiden un proceso relacional para apoyar los procesos de enseñanza y aprendizaje. Por ejemplo, se señala una baja tolerancia a la frustración, baja motivación y en algunos casos la normalización de violencia física y verbal de algunos estudiantes.

se ven problemas de violencia y hay tantos problemas de convivencia y en los mismos cursos que de repente eso igual frustra profesionalmente el trabajo que uno viene a hacer...la normalización de actitudes que tienen en la casa, porque ellos tienen normalizado no sé, decir garabatos son muy groseros, emm las actitudes violentas que dije anteriormente y contrarrestar eso de repente resulta muy difícil. (P2, $\mathrm{EF)}$

les falta motivación, mucha motivación, no están motivados, ellos quieren la nota no más, pero yo les digo "ustedes están acá, porque tienen que aprender, no por una nota" Ni si quiera lo intentan a veces, entonces, es algo con lo que tengo que lidiar todos los días, me dicen "no es que eso es muy difícil". (Li_P2)

Por otra parte, al comparar sistemas educativos, las familias migrantes no aprecian grandes diferencias entre la escuela pública en Venezuela o en Chile, señalan las mismas formas de contacto (cuaderno de notas, reuniones de apoderados, wasap de curso) y en cuanto a contenidos, también refieren cercanía entre curriculums.

tenemos a las niñas en colegios municipalizados, la educación que recibe esta buena, a pesar de todo el bombardeo que tengo, creo que hay como una especie de fantasma que persigue aquí con respecto a la educación pública o privada, pues yo que tengo la oportunidad de reunirme con los compañeros, colegas de mi esposo y veo a su hijo y veo a mi hija, creo que el nivel de conocimiento está allí. (F1_M)

Por último, en uno de los establecimientos el profesorado menciona frecuentemente la falta de apoyo de las familias locales en el proceso educativo de sus hijos. Por ejemplo, se describen inasistencias por parte de padres y apoderados a actividades de encuentro como las reuniones de curso, talleres, reuniones específicas con estudiantes o actividades de recreación promovidas en el establecimiento.

yo tengo 42 en mi curso, llegan 12 papás, 15 y eso ya es como lo máximo y son los mismos de siempre, ..., ellos mismos dicen "queremos hacer algo bonito para nuestros hijos y al final los que terminan reclamando, son los que no vienen a reunión", y eso pasa. (P2, Li)

\section{Discusión}

Como plantea Moll y otros (1992) el objetivo de los FdC es "desarrollar innovaciones curriculares basadas en el conocimiento y habilidades de familias migrantes de clase trabajadora basadas en la observación de los hogares, así como en la sala de clases" (p. 132). El presente artículo da cuenta de las prácticas de los hogares de familias migrantes, sus valores y actitudes frente a la educación, así como de las prácticas del día a día en la sala de clases desde la experiencia de los propios docentes, relevando así el contexto en el cual se encuentran el hogar y la escuela. Los resultados sugieren, por una parte, sofisticados FdC de las familias venezolanas participantes de este estudio, así como una clara continuidad entre los FdC, considerando las expectativas, actitudes y comportamientos esperados en la escuela. 
La continuidad con la escuela, la coordinación y comunicación con ésta, se muestra posible en la medida que estas familias han internalizado transgeneracionalmente, ciertas convenciones sociales en relación a cómo funcionar, relacionarse y comportarse dentro de la cultura escolar y, en coherencia con el habitus de clase media, como señala Bourdieu (1986), o un marco de referencia cultural como características primarias (Ogbu, 1987) que las han llevado a adoptar significados compartidos en relación a la educación formal, favoreciendo la integración escolar en el nuevo país. Más específicamente, la continuidad parece estar directamente vinculada con procesos de enseñanza-aprendizaje que dan cuenta de familias migrantes poseedoras de un fuerte capital cultural, en tanto todos los entrevistados son profesionales y vienen de familias donde se promueve el logro de metas educativas de nivel superior. Las familias, con sus particularidades, promueven valores asociados a la institución escolar (Ratner, 2013), donde la autonomía, el esfuerzo, la responsabilidad personal y el respeto a las figuras de autoridad, aspectos claves para acceder a mayores oportunidades de desarrollo y promoción social de cara a un proyecto futuro.

Integrado al análisis, las entrevistas grupales realizadas en ambos establecimientos educacionales ayudan a visualizar el escenario de esta investigación desde la perspectiva de los docentes. Los resultados también muestran que éstos ejercen su profesión en contextos complejos. Resulta preocupante la presencia de violencia física y verbal reportada en la sala de clases, todo lo cual impacta el clima de aula y el desarrollo de objetivos de aprendizaje, lo cual coincide con otras investigaciones a nivel nacional (por ej. López et al., 2012). Los resultados también muestran preocupación por parte de los docentes respecto a la falta de atención, perseverancia, esfuerzo y motivación observada en estudiantes locales. La evidencia sugiere que los estudiantes chilenos que acuden a dichas escuelas están más expuestos a contextos sociales vulnerables y precarizados, con presencia de violencia en el barrio. Esto difiere con los cinco casos venezolanos incluidos en el estudio, cuyos lugares de residencia y entorno familiar no reportan dichas características.

Los profesores en general expresan una falta de compromiso de las familias locales con el proceso educativo de los hijos/as, tanto en instancias formales propias de la escuela (por ej. asistencia a reuniones), como en actividades organizadas para fortalecer otras áreas del ámbito escolar como paseos de curso, convivencias o fiestas. Situación contraria en el caso de las familias migrantes venezolanas donde el apoyo e implicación parental en las rutinas escolares es alto y se observa que adoptan formas de prácticas guiadas en el hogar. Éstas en palabras de Rogoff, "ponen foco en el sistema interpersonal de compromisos y disposiciones... al promover cierto tipo de involucramiento y restringir otros" (Rogoff, 1995, p. 146). En este caso se observa que las actividades se encuentran encaminadas a alcanzar acreditación escolar y ascender en una carrera académica.

Por tanto, los resultados anteriores dan cuanta de una alta valoración del profesorado hacia las familias migrantes que llegan a sus aulas en escuelas públicas situadas en el sur de Chile, resultados que contrastan con investigaciones realizadas en el norte del país en contextos educativos similares. Esta discrepancia podría relacionarse, por un lado, a los referentes de raza negra, indígena y/o mestiza que la población migrante venida de países fronterizos presenta en el norte del país, configurado histórica y socialmente estereotipos culturales en forma de racismo y xenofobia, como señala la evidencia (Pavez, 2012; Tijoux, 2013). Por otra parte, esta alta valoración puede estar asociada al papel que juegan familias con las características descritas, dentro del modelo educativo chileno, por cuanto aportan altos resultados académicos a las escuelas, lo que les favorece en un sistema de rendición de cuentas por desempeño escolar (Falabella y De la Vega, 2016), a la vez que aportan en la mejora del clima de aula con repertorios de conducta deseables y esperados entre el estudiantado.

\section{Conclusiones}

Los resultados anteriores sugieren por un lado un hecho evidente desde la perspectiva sociocultural, cual es la interdependencia inevitable entre procesos de aprendizaje-enseñanza, los contextos históricos y culturales, las realidades de los hogares y los factores macro culturales que rigen a las instituciones escolares. Como señala Bronfenbrenner (1985) "nuestra ciencia es particularmente unidireccional. Sabemos mucho más de los niños que de los entornos en los que viven o de los procesos mediante los cuales esos entornos afectan al curso del desarrollo" (p. 45). En esta línea, la investigación sugiere la necesidad de explorar las realidades de los hogares y las formas en las que es valorada (o no) la enseñanza-aprendizaje 
escolar en las familias locales. Esto es clave para integrar a los distintos actores del sistema incluyendo la mirada docente, la escuela, el estudiantado, así como también a las familias y su lugar de procedencia. Esto último, refiriéndose a la importancia de buscar medios alternativos de vinculación con las familias, más allá de las medidas tradicionales que, en la actualidad, se muestran poco coherentes con, por ejemplo: la diversidad de las familias y sus circunstancias; las extensas jornadas de trabajo de los padres y madres; las identidades biográficas; o las variadas estructuras familiares, entre otras.

El uso de los FdC como aproximación a la pregunta de investigación permitió vislumbrar también la variabilidad en las características de los migrantes y los procesos migratorios que se insertan en espacios educativos en Chile. Se focalizó la atención en escuelas situadas en entornos vulnerables, tal como lo han hecho los estudios pioneros en la materia, sin embargo, los resultados indican la presencia de FdC ligados fielmente a la tradición escolar, lo que paradójicamente hace pensar en incluir a las familias locales dentro de la exploración y validación de sus propios FdC en futuras investigaciones. En este sentido, el concepto de justicia social pone en evidencia la necesidad de atender también a los contextos en que los estudiantes locales se están desarrollando, más allá de la precariedad y conflictos psicosociales con los que se les vincula. Investigaciones en Chile sugieren un panorama que requiere ser revisado.

Otro de los aspectos relevantes es la alta valoración que las familias migrantes hacen de la educación pública nacional que, tras décadas de la instalación de las lógicas del libre mercado en su dinámica y estructura, ha generado un deterioro paulatino en su calidad e imagen. Estudios previos ya muestran que son precisamente las familias migrantes las que aportan en el aumento de la matrícula pública. Todo lo anterior genera altas expectativas respecto al aprovechamiento y valor que esta nueva diversidad promueve en los entornos escolares más vulnerables. Por último, es importante señalar que los resultados deben ser consideradas con precaución, dadas las características de la muestra y de la diferencia en proporción entre número de estudiantes migrantes y número de estudiantes chilenos en el aula incluidos en la muestra, lo cual es propio del contexto de investigación no experimental en el cual se inserta esta investigación.

\section{Agradecimientos}

La investigación se desarrolló gracias al proyecto "Rescatando los Fondos de Conocimiento de las familias como estrategia de vinculación con la escuela. Repensando el aula desde el aprendizaje situado" (DID Regular S-2018-13), financiado por la Universidad Austral de Chile. Agradecemos también a la comunidad educativa y a las familias migrantes que nos recibieron durante el transcurso de la investigación.

\section{Referencias}

Bellei, C. (2010). Evolución de las políticas educacionales en Chile (1980-2009). En A. Bilbao y A. Salinas. El libro abierto de la informática educativa. Leccionesy desafíos de la Red Enlaces (pp.14-34). Ministerio de Educación Chile.

Biscarra, C., Giaconi, C. y Assaél, J. (2015). El docente en la legislación educacional chilena. Psicoperspectivas, 14(3), 80-92. https://doi.org/10.5027/psicoperspectivas-Vol14-Issue3-fulltext-600

Bourdieu, P. (1986). The forms of capital. En R. J (Ed.), Handbook of theory and research for the sociology of education (pp. 241-258). Greenwood.

Bronfenbrenner, U. (1985). Contextos de crianza del niño. Problemas y prospectiva. Infancia y Aprendizaje, 8(29), 4550. https://doi.org/10.1080/02103702.1985.10822058

Cerón, L., Pérez, M. y Poblete, R. (2017). Percepciones docentes en torno a la presencia de niños y niñas migrantes en escuelas de Santiago: Retos y desafíos para la inclusión. Revista Latinoamericana de Educación Inclusiva, 11(2), 233-246. https://doi.org/10.4067/S0718-73782017000200015

Cole, M., Packer, M. y Kobelt, E. (2014). Designing for diversity and inclusion: The fifth dimension. Psicologia, Conocimiento y Sociedad, 4(2), 28-61.

Contreras-Cruces, H. (2016). Migraciones locales y asentamiento indígena en las estancias españolas de Chile central, 1580-1650. Historia, 49(1), 87-110. https://doi.org/10.4067/S0717-71942016000100004 
Duconge, G., Guizardi, M. (2014). Afroariqueños: Configuraciones de un proceso histórico de presencia. Estudios Atacameños, 49, 129-151. https://doi.org/10.4067/S0718-10432014000300008

Esteban-Guitart, M., Oller, J. y Vila, I. (2012). Vinculando escuela, familia y comunidad a través de los fondos de conocimiento e identidad. Un estudio de caso con una familia de origen marroquí. Revista de Investigación en Educación, 10, 21-34.

Esteban-Guitart, M. y Saubich, X. (2013). La práctica educativa desde la perspectiva de los fondos de conocimiento e identidad. Teoría Educativa, 25(2) 189-211. https://doi.org/10.14201/11583

Falabella, A. y De la Vega, L. (2016). Políticas de responsabilización por desempeño escolar: Un debate a partir de la literatura internacional y el caso chileno. Estudios Pedagógicos, 42(2), 395-413, https://doi.org/10.4067/S0718-07052016000200023

Fardella, C. (2013). Resistencias cotidianas en torno a la institucionalización del modelo neoliberal en las políticas educacionales: El caso de la docencia en Chile. Psicoperspectivas, 12(2), 83-92.

González, N. y Moll, L. C. (2002). Cruzando el puente: Building bridges to funds of knowledge. Educational Policy, 16(4), 623-641. https://doi.org/10.1177/0895904802016004009

Gubbins, V. y Otero, G. (2016). Efecto de la rendición de cuentas de las escuelas chilenas sobre la participación de padres. Revista de Educación, 372, 9-34. https://doi.org/10.4438/1988-592X-RE-2015-372-313

Iglesias, E., Patiño-Gonzáles, J., Lalueza, J. L. y Esteban-Guitart, M. (2020). Manifiesto en Tiempos de Pandemia. Por una Educación Crítica, Intergeneracional, Sostenible y Comunitaria. Revista Internacional de Educación para la Justicia Social, 9(3e), 181-198. https://doi.org/10.15366/riejs2020.9.3.010

Instituto Nacional de Estadísticas y Departamento de Extranjería y Migración. (2020). Estimación de personas extranjeras residentes en Chile. 31 de diciembre 2019. Chile.

Jiménez, F. y Fardella, C. (2015). Diversidad y rol de la escuela. Discursos del profesorado en contextos educativos multiculturales en clave migratoria. Revista Mexicana de Investigación Educativa, 20(65), 419-41.

Johnson-Mardones, D. (2017). Investigación cualitativa y educación: tensiones en su propuesta, desarrollo, escritura y publicación. Enfermería: Cuidados Humanizados, 6, 83-88. https://doi.org/10.22235/ech.v6iespecial.1455

Joiko, S. y Vásquez, A. (2016). Acceso y elección escolar de familias migrantes en Chile: No tuve problemas porque la escuela es abierta, porque acepta muchas nacionalidades. Calidad en la Educación, 45, 132-173. https://doi.org/10.4067/S0718-45652016000200005

Lalueza, J. (2012). Modelos psicológicos para la explicación de la diversidad cultural. Cultura y Educación, 24 (2), 149 162. https://doi.org/10.1174/113564012804932119

López-Calva, J. (2019). Ética e investigación educativa: Aproximación teórica para su comprensión desde la estructura dinámica del bien humano. Revista Colombiana de Educación, 76, 223-242.

López, V., Bilbao, M. de los A. y Rodríguez, J. I. (2012). La sala de clases sí importa: Incidencia del clima de aula sobre la percepción de intimidación y victimización entre escolares. Universitas Psychologica, 11(1), 91-101. https://doi.org/10.11144/Javeriana.upsy11-1.scii

López Reyes, E. A., Juárez Hernández, L. G. y Veytia Bucheli, M. G. (2019). Typological scheme of migration and forced displacements. Estudios Frontizeros, 20, 1-21. https://doi.org/10.21670/ref.1907028

Llopart, M. y Esteban-Guitart. M. (2016). Funds of knowledge in 21st century societies: inclusive educational practices for under-represented students. A literature review. Journal of Curriculum Studies, 50(2), 145-161. https://doi.org/10.1080/00220272.2016.1247913

Marolla, J. (2019). La difícil tarea de la interculturalidad desde la didáctica de las ciencias sociales. Análisis comparativo de los programas de Argentina, Colombia y Chile. El Futuro del Pasado, 10, 159-186. https://doi.org/10.14516/fdp.2019.010.001.006

Ministerio de Educación. (2018). Política nacional de estudiantes extranjeros 2018-2022. MINEDUC.

Moll, L. C., Amanti, C., Neff, D. y Gonzalez, N. (1992). Funds of knowledge for teaching: Using a qualitative approach to connect homes and classrooms. Theory into Practice, 31,132-141. https://doi.org/10.1080/00405849209543534 
Nascimento, J., Meireles, I., Ribeiro, M., Braga, T., Catafesta, F. y Bernardino, E. (2016). Uso del software NVivo ${ }^{\circledR}$ en una investigación con teoría fundamentada. Index de Enfermería, 25(4), 263-267.

Ogbu, J. U. (1987). Variability in minority school performance. A probleme in search of an explanation. Anthropology and Education Quarterly, 18, 312-334. https://doi.org/10.1525/aeq.1987.18.4.04x0022v

Pavez, I. (2012). Inmigración y racismo: Experiencias de la niñez peruana en Santiago de Chile. Revista de Estudios Transfronterizos, 12(1), 75-99. https://doi.org/10.4067/S0719-09482012000100004

Peña-Cuadra, M. y Esteban-Guitart, M. (2013). El estudio de las identidades desde un enfoque cualitativo. La multimetodología autobiográfica extendida y los talleres lúdico-reflexivos. EMPIRIA. 26, 175-200. https://doi.org/10.5944/empiria.26.2013.7157

Poveda, D. (2001). La educación de las minorías étnicas desde el marco de las continuidades-discontinuidades familia-escuela. Gazeta de Antropología, 17, 17-31. https://doi.org/10.30827/Digibug.7491

Ratner, C. (2013). Desde Vygotski a la psicología macrocultural. Documenta Universitaria.

Riedemann, A. y Stefoni, C. (2015). Sobre el racismo, su negación, y las consecuencias para una educación anti-racista en la enseñanza secundaria chilena. Polis, 14(42), 191-216. https://doi.org/10.4067/S0718-65682015000300010

Rogoff, B. (1995). Observing sociocultural activity on three planes: Participatory appropriation, guided participation, and apprenticeship. En J. V. Werstch, P. del Río, y A. Álvarez (Eds.), Sociocultural Studies of Mind (pp. 157187). Cambridge University Pres.

Rogoff. (2003). The cultural nature of human development. Oxford University Press

Rogoff, B. y Lave, J. (1984). Everyday cognition: Its development in social context. Harvard University Press.

Sandoval, E. y Lamas, M. (2017). Impacto de la Ley SEP en las escuelas. Una mirada critica y local en torno al rol de los psicólogos de la educación. Paideia, 61, 57-81.

Scribner, S., y Cole, M. (1973). Cognitive consequences of formal and informal education. Science, 182(4112), 553-559. https://doi.org/10.1126/science.182.4112.553

Stake, R. E. (1995). The Art of Case Study Research. Sage Publications.

Tijoux, M. (2013). Niños (as) marcados por la inmigración peruana: Estigma, sufrimientos y resistencias. Convergencia Revista de Ciencias Sociales, 61, 83-104.

Vila, I. (1998). Familia, escuela y comunidad. Horsori.

Villalobos, A., Sanhueza, S. y Norambuena, C. (2017). Migración y territorialidad: Aportes para la construcción de modelos educativos interculturales. Papeles de trabajo, Centro de Estudios Interdisciplinarios en Etnolingüística y Antropología Socio-Cultural, 33, 92-109.

\section{Breve CV de las autoras}

\section{Macarena Lamas Aicón}

Profesora auxiliar del Instituto de Estudios Psicológicos de la Universidad Austral de Chile (UACh), Magíster en Psicología de la Educación de la Universidad de Barcelona y Doctora en la misma área de la Universidad Autónoma de Barcelona. Sus principales líneas de investigación son: inclusión social y educativa del alumnado migrante; el estudio de procesos de innovación educativa para la mejora de las prácticas escolares en clave inclusiva; continuidades y discontinuidades entre familia y escuela. Actualmente es la investigadora principal del proyecto DID Regular S-2018-13 que ha originado este trabajo.

Email: macarena.lamas@uach.cl

ORCID ID: https://orcid.org/0000-0002-2340-4249 


\section{Patricia Thibaut}

Ph.D en Educación de la Universidad de Sydney. Académica del Instituto de Historia y Ciencias Sociales de la Universidad Austral de Chile. Sus intereses de investigación abarcan el nexo entre aprendizaje, literacidad y tecnología en espacios formales e informales. Ha dirigido el proyecto Fondecyt $\mathrm{N}^{\circ} 3160114$ "Exploring the nexus between literacy, technology and classroom practices in the Chilean context" es co-investigadora del Proyecto DID Regular S-2018-13 que ha originado este trabajo y actualmente lleva a cabo la investigación "Oportunidades de habla en el aula: Continuidad y cambio en la enseñanza" (FIDUACh). Email: patricia.thibaut@uach.cl

ORCID ID: https://orcid.org/0000-0003-2990-0339 